\title{
Neurophysiologic Markers of Abnormal Brain Activity in Schizophrenia
}

\author{
Anthony J. Rissling • Scott Makeig • David L. Braff • \\ Gregory A. Light
}

Published online: 21 September 2010

(C) The Author(s) 2010. This article is published with open access at Springerlink.com

\begin{abstract}
Cortical electrophysiologic event-related potentials are multidimensional measures of information processing that are well-suited for efficiently parsing automatic and controlled components of cognition that span the range of deficits evidenced in schizophrenia patients. These information processes are key cognitive measures that are recognized as informative and valid targets for understanding the neurobiology of schizophrenia. These measures may be used in concert with the Measurement and Treatment Research to Improve Cognition in Schizophrenia (MATRICS) neurocognitive measures in the development of novel treatments for schizophrenia and related neuropsychiatric disorders. The employment of novel event-related potential paradigms designed to carefully characterize the early spectrum of perceptual and cognitive information processing allows investigators to identify the neurophysiologic basis of cognitive dysfunction in schizophrenia and to examine the associated clinical and functional impairments.
\end{abstract}

A. J. Rissling • D. L. Braff · G. A. Light $(\bowtie)$

Department of Psychiatry, University of California San Diego, 9500 Gilman Drive, La Jolla,

San Diego, CA 92093-0804, USA

e-mail: glight@ucsd.edu

A. J. Rissling

e-mail: ajrissling@ucsd.edu

D. L. Braff

e-mail: dbraff@ucsd.edu

S. Makeig

Institute for Neural Computation,

University of California San Diego,

9500 Gilman Drive \#0559, La Jolla,

San Diego, CA 92093-0559, USA

e-mail: smakeig@ucsd.edu
Keywords Schizophrenia Event-related potentials . Mismatch negativity $\cdot$ P300 $\cdot$ Reorienting negativity Functional outcome

\section{Introduction}

Navigation through stimulus-laden and psychosocial environments requires the adaptive and integrative processing of a nearly infinite number of sensory inputs. Successful information processing requires efficiency at preattentive (automatic) levels of information processes in order to screen out or inhibit responses to redundant or irrelevant inputs and, reciprocally, to automatically allocate finite processing resources to novel and/or salient stimuli. Appropriate screening and further processing of information is dependent on an efficient balance with these core and automatic levels of processing; this process typically occurs outside of awareness with flexibility in crucial neural substrates that allows for conscious and near-conscious allocation of resources and responses toward potentially salient information. In this context, schizophrenia patients evidence abnormalities at automatic as well as controlled levels of sensory and higher-order cognitive information processes [1]. An emerging view is that the commonly observed integrative higher-order cognitive deficits in schizophrenia that in turn contribute to psychosocial disability emerge, at least in part, from dysfunction in the coordination of neural activity at the preattentive and early attentive levels of information processing. Indeed, these early levels of sensory processing are viewed as very useful biomarkers of drug response and outcome. These core functions may relate to important demographic, clinical, neuropsychological, and functional outcome variables $[2,3]$ and thus provide a useful platform for exploring the 
underlying neural substrates and genetic architecture that support these functions $[4,5 \cdot \bullet]$.

Event-related potentials (ERPs) are temporally precise measures of information processing that are well-suited to separately measure the automatic and controlled components of cognition that are deficient in schizophrenia patients. The auditory oddball task is the prototypical experimental paradigm that is used to elicit automatic sensory-based components through attention-dependent and decision-related responses. In this paradigm, a sequence of frequently presented (eg, 90\%) standard sounds are occasionally interrupted (eg, 10\%) by deviant or "oddball" stimuli. Examples of these stimuli include sounds that differ from standard stimuli in their duration, pitch, or intensity. For nearly 50 years, the oddball paradigm has proven to be a very versatile tool in testing normal and deficient information processing because different features of the ERP waveform following standard and deviant stimuli yield important information. Depending on the stimulus and/or task parameters, a sequence of ERP components that vary in latency, amplitude, and scalp distribution are elicited. These components have been informative for revealing normal and abnormal information processing associated with underlying neural substrates (Fig. 1).

Peaks in ERP average waveform (components) following repeated presentations of sensory stimuli index certain aspects of important progressive cascades of neural processes. These begin with early automatic sensory processes and proceed through controlled decision- and response-related processes, including the following: sensory registration (N1), automatic change detection (mismatch negativity $[\mathrm{MMN}]$ ), the orienting or covert shift in attention (P3a), allocation of attentiondependent processes (P3b), and the reorienting of attention (RON) following salient or distracting stimuli (Fig. 1). The amplitude and latency of the respective peaks are often interpreted as estimating with millisecond-level precision the strength of the allocated processing resources and the time course of their allocation. The timing of the ERP peaks across latency ranges varies with many factors, including the individual's mental state, attentional demands, processing resources, and susceptibility to distraction by irrelevant stimuli. Moreover, the distribution of voltage over the scalp in highdensity electroencephalogram (EEG) recordings, in conjunction with supplementary neuroimaging techniques, can be used to elucidate the neuroanatomic loci of these processes.

The employment of novel ERP paradigms, designed to carefully characterize the early spectrum of perceptual and cognitive information processing, has allowed investigators to identify the neurophysiologic representation of attentional dysfunction in schizophrenia and to examine the potential downstream neurocognitive, clinical, and functional factors associated with abnormalities in this early sensory and perceptual auditory information processing.

\section{Mismatch Negativity}

To measure controlled attention-dependent processes during an oddball paradigm, an active task is used in which a participant is instructed to pay attention to the stimulus stream and respond covertly or overtly to one stimulus while ignoring other standard or distracter stimuli. The participant is required to distinguish the infrequent target
Fig. 1 Idealized event-related potential waveforms representing stages of information processing that shift from automatic to higher-order controlled processes. Note the logarithmic latency scale. These waveforms cannot be elicited from a single paradigm and are thus schematic representations. $M M N$ mismatch negativity; $R O N$ reorienting of attention. (Adapted from Picton et al. [45].)

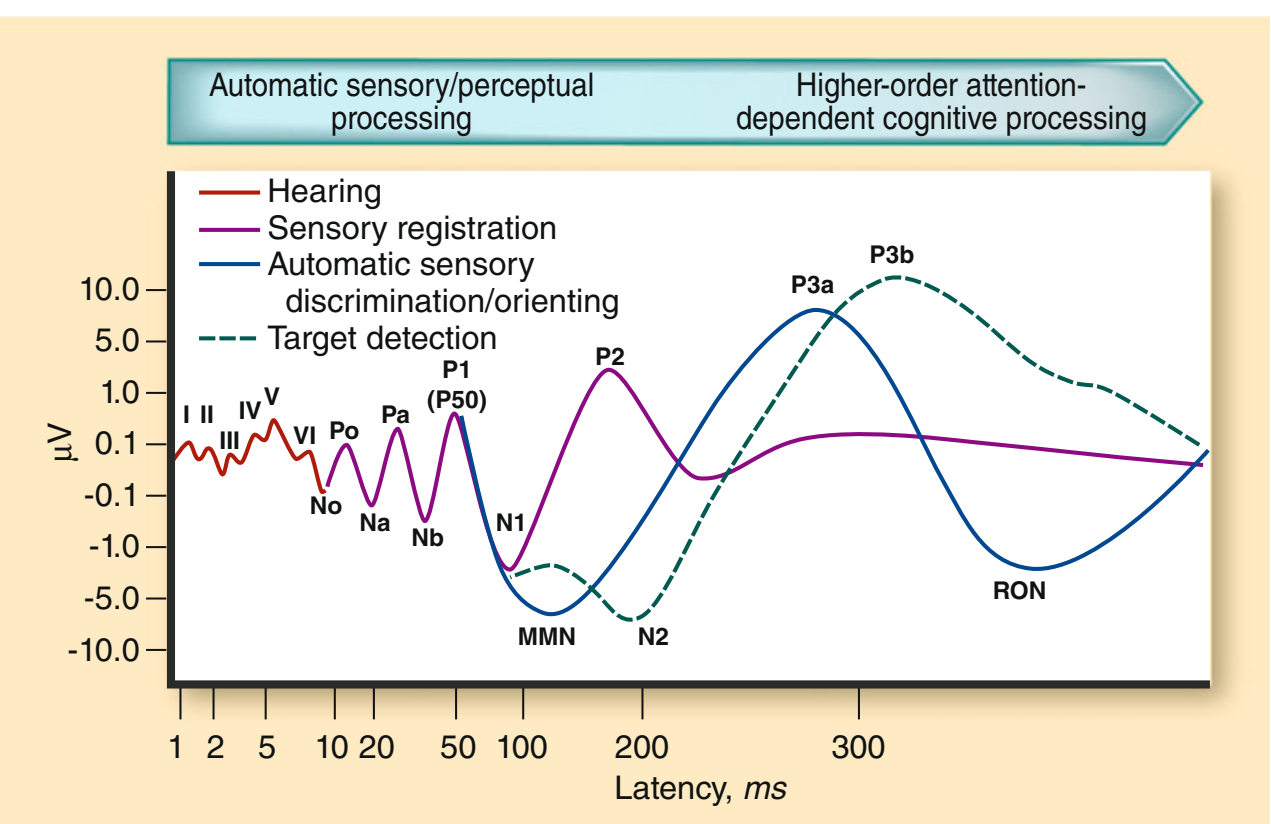


stimulus from the frequent standard stimulus by noting the occurrence of the target, typically by pressing a button or mentally counting. In contrast to the active oddball task, the passive version does not require a behavioral response from the participant. In this context, the individual's attention is often directed away from the sequence of standard and deviant tones toward another moderately demanding task, usually in a different modality (eg, the participant is instructed to read, watch a silent video, or even perform a difficult visual continuous performance task).

Passive oddball ERP paradigms allow investigators to interrogate the earliest stages of information processing free of attentional and motivational artifacts that may confound the assessment of some higher cognitive operations in clinical populations, including schizophrenia patients [4]. In a classic auditory oddball paradigm, a duration-deviant stimulus elicits a modality-specific MMN peak 100 to $200 \mathrm{~ms}$ after the onset of a stimulus deviance [6]. As MMN can be elicited even when participants do not attend to the stimuli, it is assumed to reflect a preattentive deviance detection process [7]. MMN reflects an important process that automatically detects stimuli. Physiologically, the MMN is the first measurable brain response component that differentiates between standard and deviant auditory stimuli [8], as no MMN component is elicited by the repeated standard stimuli.

Deficits in MMN generation represent a remarkably robust finding in schizophrenia. Several published reports of reduced MMN in schizophrenia patients have reported varying stimulation parameters (eg, pitch, duration, and intensity) and conditions $[9,10 \bullet, 11]$. In a meta-analysis carried out by Umbricht and Krljes [12], the mean effect size of the schizophrenia deficit was about $1.0 \mathrm{SD}$, a large deficit by common standards.

Many cross-sectional studies of MMN in healthy individuals indicate an age-related diminution of MMN $[13,14]$. Importantly, our recent study of 250 schizophrenia patients and 150 nonpsychiatric comparison individuals demonstrated that schizophrenia patients still exhibit an approximately 1.0-SD effect size deficit across the adult age range [10•]. Future studies, currently in progress with colleagues, will expand these results to the examination of neurophysiologic predictors of cognitive and health factors in geriatric schizophrenia patients and matched controls.

MMN deficits in schizophrenia patients are highly associated with patients' impairments in daily functioning, level of independence in their community-living situation, and functional outcome $[3,15 \bullet, 16-19,20 \bullet]$. Across studies, schizophrenia patients with more severe functional impairments had smaller (ie, less negative) MMNs than higher-functioning patients. Tone-duration MMN deficits in schizophrenia patients were highly associated $(r=0.65)$ with clinician ratings of global daily functioning in 25 chronic schizophrenia patients, and the $\mathrm{MMN} /$ function correlation had the same frontocentral topographic distribution as the MMN deficits in schizophrenia patients. That is, the scalp distribution of MMN deficits in schizophrenia patients across electrodes was strikingly similar to the topographic pattern of correlation between MMN amplitude and functional impairment within the schizophrenia patient group.

A related study of patients longitudinally assessed during a 15-month interval confirmed that the MMN deficits in schizophrenia were highly associated with ratings of functional status at both first $(r=0.63)$ and second $(r=0.68)$ test sessions, indicating that the MMN/function relationship is stable even across relatively long time intervals [19]. While several studies have determined that attention-dependent neurocognitive tests are modestly associated with functioning in schizophrenia patients (eg, with correlations of $r=0.30$ to $r=0.40$, accounting for $9 \%$ to $16 \%$ of the variance), MMN deficits correlate with functioning up to $r=0.68$, accounting for up to $46 \%$ of the variance in functional status [3, 19].

This association of MMN and functional deficits in schizophrenia patients has been replicated and extended by an independent research group. Kawakubo et al. [18] reported a relationship between functional deficits and MMN in response to a duration change of Japanese speech sounds $(r=0.53)$. Other studies of schizophrenia patients have found that MMN is associated with verbal memory deficits [18], executive functioning [17], and degree of social skills acquisition following a 3-month training program [21•]. Significant associations between MMN and the Global Assessment of Functioning scale scores also have been noted in healthy individuals [22].

A very recent study by Wynn et al. [15•] extended the literature by examining correlations among MMN amplitude, daily functioning, and social cognition (social perception and theory of mind) in schizophrenia patients. Examining MMN, social cognition, and four domains of functioning (work, independent living, social networks, and family networks) in schizophrenia patients and healthy controls, Wynn et al. [15•] first replicated the results of Light and Braff [3, 19], reporting that schizophrenia patients exhibited reduced MMN activity at frontocentral electrode sites compared with healthy controls and, second, reported that a larger MMN at frontocentral sites correlated with better functioning at work $(r s=0.37-0.45)$, independent living $(r s=0.37-0.51)$ (although not in social or family networks), and with better social perception $(r s=0.36-0.50)$. The results suggest that MMN activity may be more closely tied to some outcome domains (eg, work and independent living) than others.

Employing high-resolution structural magnetic resonance scans to generate group average cerebral cortex and temporal lobe models using cortical pattern matching, Rasser et al. [16] 
investigated whether the relationship between reduced MMN and impaired daily functioning is mediated by cortical volume loss in temporal and frontal brain regions in schizophrenia, and whether this relationship varies with the type of auditory deviant that generates the MMN. They reported that MMN amplitude was reduced in schizophrenia patients and correlated with their impairment in day-to-day function as rated on the Social and Occupational Functioning Assessment Scale. In the schizophrenia patients, bilateral gray matter reduction in Heschl's gyrus as well as motor and executive regions of the frontal cortex was correlated with reduced MMN amplitude in response to frequency deviants, while reduced gray matter in right Heschl's gyrus was also correlated with reduced MMN to duration deviants. The results link frontotemporal cerebral gray matter pathology to MMN amplitude and daily functioning in schizophrenia patients.

\section{MMN Transition to P3a}

The MMN is often followed by the P3a, a frontocentral positivity peaking at about $300 \mathrm{~ms}$ from the onset of the deviant stimulus. $\mathrm{P} 3 \mathrm{a}$ is assumed to reflect the covert orienting or shift in attention [23, 24]. Several studies have found P3a amplitude in response to infrequent nontarget or distracter stimuli to be decreased in schizophrenia patients [3, 9, 15• 16-19, 25, 26]. Light et al. [22], employing a duration-deviant $\mathrm{MMN} / \mathrm{P} 3 \mathrm{a}$ paradigm, reported across frontocentral electrode regions that significant correlations were observed between psychosocial functioning and MMN $(r=0.62)$ and P3a $(r=0.63)$ amplitudes in healthy individuals. P3a amplitude was also associated with immediate and delayed recall of verbal information, with significant correlations widely distributed across frontocentral recording areas $(r=0.72)$. The latency of the $\mathrm{P} 3 \mathrm{a}$ response was significantly associated with working memory performance $(r=0.53)$ and functional ratings $(r=0.48)$.

Recent evidence suggests associations between MMN and P3a amplitude deficits, with higher-order cognitive and psychosocial functioning possibly marking the beginning of progressive underlying changes that begin at illness onset. Hermens et al. [20•] reported that first-episode schizophrenia patients showed significantly reduced $\mathrm{MMN}$ and P3a amplitudes at frontal and central sites compared with controls. A decrease in P3a peak amplitude corresponded to poorer ratings on social and occupational functioning ( $r s=$ $0.49-0.64)$ and on four neuropsychological measures ( $r s=$ 0.45-0.54). MMN peak amplitude was significantly correlated with faster processing speed, simple attention $(r=0.62)$, and psychosocial functioning $(r=0.50)$.

Studies conducted in our laboratory and in collaboration with our colleagues Drs. Jahchan, Cadenhead, Braff, and Malaguti using duration-deviant stimuli to elicit the MMN suggest that small MMN reductions may be present early in the course of illness or perhaps even in individuals who are at risk of developing schizophrenia. Clearly, additional studies are necessary to delineate the nature of the MMN abnormality, its prevalence among prodromal and/or firstepisode schizophrenia patients, its utility for predicting conversion to psychosis in individuals at genetically high risk of developing the disorder, and the potential associations with clinical and functional measures.

\section{Conclusions and Future Directions}

Early preattentive sensory processes are responsible for suppressing attention-redundant or irrelevant environmental changes. These sensory processes are presumed to be elicited automatically with little or no effort, engagement, or awareness on the part of the individual $[4,27]$. When novel or salient changes occur in an ongoing auditory stream, a cascade of neural processes preattentively or automatically detects them. Depending on contextual demands, these physiologic processes then facilitate appropriate responses. Top-down processes bias stimulus selection based on criteria including salience or relevance to a goal-directed task. Although they are often viewed as discrete or dichotomous, automatic and controlled processes more likely represent a "spectrum" from automatic/preattentive to controlled/attention-dependent processes [4].

In this context, the amplitudes of MMN and P3a ERP components demonstrate an intriguing relationship between basic sensory processing and higher-order measures of learning and memory and psychosocial functioning in both healthy individuals [22] and schizophrenia patients [3, 19]. It is conceivable that the neural substrates that regulate automatic monitoring and detection of environmental changes trigger a cascade of higher-order processing. This cascade may determine whether environmental cues are salient or trivial and thus in need of further processing, allowing for inattention and suppression of further processing in accord with classic information-processing theories [28]. Efficiency at such elementary neurophysiologic levels can free up attention-dependent, controlled cognitive resources for the successful encoding, retrieval, and discrimination of task-relevant information, in turn facilitating the responsive processing necessary for adaptive cognitive and social functioning [29].

Physiologically, the MMN is the first measurable ERP component that differentiates between usual (standard) and unusual (deviant) auditory stimuli [8]. Cascade models suggest that attention-dependent decision and behavioral responses are governed by preattentional sensory memory processes elicited by the MMN. Tiitinen et al. [30] reported the latency of the MMN response predicted behavioral 
response latency and argued that attentive behavioral latency originates from preattentive sensory memory mechanisms (ie, as indexed by the MMN). Changes in processing time of sensory memory linearly transfer to subsequent perceptual, attentive, and motoric processes that are fundamental in determining appropriate behavior. Cascade models suggest that MMN deficits cascade through the orienting or covert shift in attention (indexed by P3a), allocation of attention-dependent processes (indexed by P3b), and the reorienting of attention (indexed by RON).

Using an auditory duration discrimination task, Horvath et al. [31] reported that the three processes elicited by distracting stimuli represented by the N1, MMN, P3a, and RON do not form a strongly coupled chain. Pairwise dissociations were reported between the ERPs, suggesting each processing stage can be elicited without concurrent elicitation of subsequent processes. This evidence suggests that during active attention paradigms, sensory registration (indexed by N1), automatic change detection (indexed by $\mathrm{MMN}$ ), the orienting or covert shift in attention (indexed by $\mathrm{P} 3 \mathrm{a}$ ), and the reorienting of attention (indexed by RON) may independently affect or associate with downstream neurocognitive, clinical, and functional factors. An important next step is to use novel paradigms to comprehensively characterize the profile of automatic sensory/perceptual processes, the succeeding transitions to attention-dependent processes, and their associations with neurocognitive and functional outcome in schizophrenia so as to disentangle the functionally relevant pathologies in neurocognitive processes. Current and future approaches to elucidate the underlying neural architecture of these processes include novel advances in EEG source localization and translational neurochemical modeling in rodents.

\section{Source Localization}

Source localization is a valuable tool for investigating electrical activity in the brain and offers the next step in understanding the common deficits observed in schizophrenia. It may substantially facilitate our understanding of the dynamic integration of the multiple cortical sources that likely underlie the cortical abnormalities represented by ERP differences. Typically, ERP components are assessed via waveform averaging of EEG responses to a large number of similar sensory stimuli presented during a recording session. This is followed by measurement of features of the single-channel ERP waveforms. This standard approach has proven useful for detecting important deficits in neurocognitive functioning, potential genetic endophenotypes, associations with functional outcome, and the gross scalp projections of electrical activity from the underlying neural architecture. However, standard ERP averaging and interpretation reveals little about the number, type, and spatial distribution of the sources of the relevant brain potentials.

Advances in EEG processing analyses now provide many advantages over traditional ERP averaging, allowing for the decomposition of event-related time frequency dynamics that may not be well-represented in standard response averages [32]. They also offer the ability to empirically separate the recorded responses into highresolution dissociable component processes that facilitate identification and spatial resolution of the neural sources of these responses [32-35] while retaining all the temporal resolution of traditionally measured ERP scalp recordings.

The independent component analysis (ICA) approach to dynamic brain imaging is to separate the independent EEG activities in each individual's data, not by direct spatial filtering for activities generated in a set of predefined cortical locations, but rather by using the information content of the data itself to separate portions of the recorded scalp data from each active cortical and artifact source area. This blind source separation approach carries the assumption that over sufficient recording time, the activity time courses of these processes should be nearly independent of each other and thus should contribute independent information to the multidimensional source mixtures recorded at the scalp channels.

The major advantage of the ICA approach is that the scalp potentials projecting from a single EEG source will be grouped together into a single independent component (IC) process consisting of an activity time course and fixed scalp topography, while activities of uncoupled and mainly unrelated EEG sources will be separated from this IC and isolated as summing activity projecting from other ICs. Technically, ICA finds a set of fixed spatial filters that together constitute the most distinct (ie, temporally nearindependent) signals available in the input data such that their projections to the scalp channels sum to the original data. Under favorable circumstances, ICA transforms the recorded high-density scalp data into a set of cortical brain and artifactual non-brain source waveforms, thereby discovering the temporally and information distinct signals that are contained in the data before asking directly where these signals are generated. However, the scalp map associated with each IC represents its projection to the scalp free of activities from all other sources, thus highly simplifying source localization estimation for both eventrelated and ongoing EEG activities.

In addition, statistical analysis of changes in spectral power and phase across single trials time-locked to experimental events can characterize event-related perturbations in the oscillatory dynamics in ongoing EEG signal processes. Applying such measures to the activity time courses of separated independent component sources avoids 
the confounds caused by cancellation of summing positive and negative potentials from different sources at the recording electrodes, and by misallocating to presumed locations below the recording electrodes activity that originates in various - often distant-cortical sources. In this way, the event-related spectral perturbation measure [36] may be applied to measure event-locked changes in spectral power, and intertrial phase coherence to measure the appearance and degree of this consistency near experimental events. Thus, the event-related EEG dynamics in single trials summed to form the ERP may be decomposed into constituent more or less phase-locked frequency bands, providing additional information about the relationship between the ERP and the whole EEG data.

Combining ICA preprocessing with time/frequency modeling has several benefits for cognitive neuroimaging and clinical neuroscience. ICA minimizes the influence of volume conduction and thus can be used to identify activities of individual cortical sources of clinically relevant ERP and EEG differences. The oscillatory dynamics can be more physiologically plausible, more tightly linked to behavior, and more amenable to trial-by-trial analysis than conventional ERP measures applied to scalp data [37-40]. The combined ICA time/frequency approach may prove to index brain pathophysiology in schizophrenia and other psychiatric disorders more accurately than conventional measures. An open source toolbox, EEGLAB (Swartz Center for Computational Neuroscience [University of California, San Diego]; La Jolla, CA), implementing this analysis approach is freely available (http://www.sccn.ucsd. edu/eeglab [41]).

\section{Translational Neurochemical Models}

Previous work has demonstrated that rodent ERPs share many specific similarities with humans, including mouse analogues of the N100, MMN, and P300 components that share stimulus and pharmacologic response properties with the corresponding human components [42, 43]. Neurophysiologic rodent ERP data offer unique opportunities to use pharmacologic and genetic endophenotype strategies to treat cognitive deficits in schizophrenia patients. Specifically, deviance-related ERPs have been measured in mice to model deficits in N1 and MMN to deviant tones in schizophrenia [42, 44••]. Ehrlichman et al. [42] examined the effects of the $N$-methyl-Daspartate receptor antagonist ketamine on deviance-related components and reported deviance responses were abolished by ketamine, suggesting that ketamine-induced changes in mice mimic deviance detection deficits in schizophrenia. Ehrlichman et al. [44०•] reported that in $\mathrm{Nrg}^{+/-}$mice, the MMN in response to novel stimuli was attenuated, with corresponding behavioral deficits in contextual fear conditioning and social interactions.
Acknowledgments Dr. Rissling has received grant support from and had travel/accommodations expenses covered by the National Institute of Mental Health.

Dr. Braff is partially supported by National Institute of Mental Health grant no. R01 MH065571 and by the US Department of Veterans Affairs (VISN-22) Mental Illness Research, Education, and Clinical Center.

Dr. Light has received grant support from the National Institutes of Health (no. MH079777 and VISN-22); the Mental Health Research, Education, and Clinical Center; and NARSAD.

Disclosure Dr. Light has served on a scientific advisory board for Johnson \& Johnson and as a consultant for Allergan, Memory Pharmaceuticals, and Hoffmann-La Roche. Drs. Rissling, Makeig, and Braff reported no potential conflicts of interest relevant to this article.

Open Access This article is distributed under the terms of the Creative Commons Attribution Noncommercial License which permits any noncommercial use, distribution, and reproduction in any medium, provided the original author(s) and source are credited.

\section{References}

Papers of particular interest, published recently, have been highlighted as:

- Of importance

•. Of major importance

1. Callaway E, Naghdi S: An information processing model for schizophrenia. Arch Gen Psychiatry 1982, 39:339-347.

2. Green M, Kern R, Braff D, Mintz J: Neurocognitive deficits and functional outcome in schizophrenia: are we measuring the "right stuff'? Schizophr Bull 2000, 26:119-136.

3. Light G, Braff D: Mismatch negativity deficits are associated with poor functioning in schizophrenia patients. Arch Gen Psychiatry 2005, 62:127-136.

4. Braff D, Light G: Preattentional and attentional cognitive deficits as targets for treating schizophrenia. Psychopharmacology (Berl) 2004, 174:75-85.

5. - Turetsky B, Calkins M, Light G, et al.: Neurophysiological endophenotypes of schizophrenia: the viability of selected candidate measures. Schizophr Bull 2007, 33:69-94. This comprehensive review systematically evaluated the endophenotype candidacy of key neurophysiologic abilities, including measures of inhibitory failure and impaired deviance detection. The typical experimental procedures; the current understanding of the underlying neurobiology; the nature of the abnormality in schizophrenia; the reliability, stability and heritability of the measure; and any reported gene associations were reported for each proposed index.

6. Näätänen R, Gaillard A, Mantysalo S: Early selective-attention effect on evoked potential reinterpreted. Acta Psychol (Amst) 1978, 42:313-329.

7. Näätänen R: The role of attention in auditory information processing as revealed by event-related potentials and other brain measures of cognitive function. Behav Brain Sci 1990, 13:201-288.

8. Näätänen R, Paavilainen P, Reinikainen K: Do event-related potentials to infrequent decrements in duration of auditory stimuli demonstrate a memory trace in man? Neurosci Lett 1989, 107:347-352.

9. Cortiñas M, Corral M, Garrido G, et al.: Reduced novelty-P3 associated with increased behavioral distractibility in schizophrenia. Biol Psychol 2008, 78:253-260. 
10. - Kiang M, Braff D, Sprock J, Light G: The relationship between preattentive sensory processing deficits and age in schizophrenia patients. Clin Neurophysiol 2009, 120:1949-1957. This study reported important age-related effects and considerations for $M M N$ and P3a measurement. It is the largest reported study to date in schizophrenia ( $n=253$ schizophrenia patients, 147 controls).

11. Umbricht D, Koller R, Schmid L, et al.: How specific are deficits in mismatch negativity generation to schizophrenia? Biol Psychiatry 2003, 53:1120-1131.

12. Umbricht D, Krljes S: Mismatch negativity in schizophrenia: a meta-analysis. Schizophr Res 2005, 76:1-23.

13. Cooper R, Todd J, McGill K, Michie P: Auditory sensory memory and the aging brain: a mismatch negativity study. Neurobiol Aging 2006, 27:752-762.

14. Kisley M, Davalos D, Engleman L, et al.: Age-related change in neural processing of time-dependent stimulus features. Cogn Brain Res 2005, 25:913-925.

15. - Wynn J, Sugar C, Horan W, et al.: Mismatch negativity, social cognition, and functioning in schizophrenia patients. Biol Psychiatry 2010, 67:940-947. This recent study reported that a larger $M M N$ at frontocentral sites correlated with better functioning at work, independent living, and social perception. The study extended the previous results and refined the parameters of the associations to work and independent living.

16. Rasser P, Schall U, Todd J, et al.: Gray matter deficits, mismatch negativity, and outcomes in schizophrenia. Schizophr Bull 2009 Jun 26 (Epub ahead of print).

17. Kiang M, Kutas M, Light G, Braff D: Electrophysiological insights into conceptual disorganization in schizophrenia. Schizophr Res 2007, 92:235-236.

18. Kawakubo Y, Kasai K, Kudo N, et al.: Phonetic mismatch negativity predicts verbal memory deficits in schizophrenia. Neuroreport 2006, 17:1043.

19. Light G, Braff D: Stability of mismatch negativity deficits and their relationship to functional impairments in chronic schizophrenia. Am J Psychiatry 2005, 162:1741-1743.

20. • Hermens D, Ward P, Hodge M, et al.: Impaired MMN/P3a complex in first-episode psychosis: cognitive and psychosocial associations. Prog Neuropsychopharmacol Biol Psychiatry 2010, 34:822-829. This study reported that the MMN and P3a amplitude associations with neurocognitive and functional outcome are present at the onset of schizophrenia in first-episode patients.

21. - Kawakubo Y, Kamio S, Nose T, et al.: Phonetic mismatch negativity predicts social skills acquisition in schizophrenia. Psychiatry Res 2007, 152:261-265. The study reported important results that MMN amplitude at baseline is associated with the degree of social skills acquisition following a 3-month training program.

22. Light G, Swerdlow N, Braff D: Preattentive sensory processing as indexed by the MMN and P3a brain responses is associated with cognitive and psychosocial functioning in healthy adults. J Cogn Neurosci 2007, 19:1624-1632.

23. Escera C, Alho K, Schröger E, Winkler I: Involuntary attention and distractibility as evaluated with event-related brain potentials. Audiol Neurootol 2000, 5:151-166.

24. Friedman D, Cycowicz Y, Gaeta H: The novelty P3: an eventrelated brain potential (ERP) sign of the brain's evaluation of novelty. Neurosci Biobehav Rev 2001, 25:355-373.

25. Mathalon D, Ford J, Pfefferbaum A: Trait and state aspects of P300 amplitude reduction in schizophrenia: a retrospective longitudinal study. Biol Psychiatry 2000, 47:434-449.
26. Turetsky B, Bilker W, Siegel S, et al.: Profile of auditory information-processing deficits in schizophrenia. Psychiatry Res 2009, 165:27-37.

27. Näätänen R: Attention and Brain Function: Hillsdale, NJ: Erlbaum; 1992.

28. Broadbent DE. Perception and Communication. New York: Oxford University Press; 1958.

29. Näätänen R, Tervaniemi M, Sussman E, et al.: "Primitive intelligence" in the auditory cortex. Trends Neurosci 2001, 24:283-288.

30. Tiitinen H, May P, Reinikainen K, Näätänen R: Attentive novelty detection in humans is governed by pre-attentive sensory memory. Nature 1994, 372:90-92.

31. Horváth J, Winkler I, Bendixen A: Do N1/MMN, P3a, and RON form a strongly coupled chain reflecting the three stages of auditory distraction? Biol Psychol 2008, 79:139-147.

32. Makeig S, Debener S, Onton J, Delorme A: Mining event-related brain dynamics. Trends Cogn Sci 2004, 8:204-210.

33. Anemüller J, Sejnowski T, Makeig S: Complex independent component analysis of frequency-domain electroencephalographic data. Neural Netw 2003, 16:1311-1323.

34. Makeig S, Delorme A, Westerfield M, et al.: Electroencephalographic brain dynamics following manually responded visual targets. PLOS Biol 2004, 2:e176.

35. Onton J, Westerfield M, Townsend J, et al.: Imaging human EEG dynamics using independent component analysis. Neurosci Biobehav Rev 2006, 30:808-822.

36. Makeig S: Auditory event-related dynamics of the EEG spectrum and effects of exposure to tones. Electroencephalogr Clin Neurophysiol 1993, 86:283-293.

37. Stettler DD, Das A, Bennett J, Gilbert CD: Lateral connectivity and contextual interactions in macaque primary visual cortex. Neuron 2002, 36:739-750.

38. Makeig S, Jung TP, Bell AJ, et al.: Blind separation of auditory event-related brain responses into independent components. Proc Natl Acad Sci U S A 1997, 94:10979-10984.

39. Makeig S, Westerfield M, Jung TP, et al.: Functionally independent components of the late positive event-related potential during visual spatial attention. J Neurosci 1999, 19:2665-2680.

40. Makeig S, Enghoff S, Jung TP, Sejnowski TJ: A natural basis for efficient brain-actuated control. IEEE Trans Rehabil Eng 2000, $8: 208-211$.

41. Delorme A, Makeig S: EEGLAB: an open source toolbox for analysis of single-trial EEG dynamics including independent component analysis. J Neurosci Methods 2004, 134:9-21.

42. Ehrlichman R, Maxwell C, Majumdar S, Siegel S: Devianceelicited changes in event-related potentials are attenuated by ketamine in mice. J Cogn Neurosci 2008, 20:1403-1414.

43. Siegel S, Connolly P, Liang Y, et al.: Effects of strain, novelty and NMDA blockade on auditory-evoked potentials in mice. Neuropsychopharmacology 2003, 28:675-682.

44. •- Ehrlichman R, Luminais S, White S, et al.: Neuregulin 1 transgenic mice display reduced mismatch negativity, contextual fear conditioning and social interactions. Brain Res 2009, 1294:116-127. The study is a recent demonstration of a mouse analogue to the human MMN component in mice. The translational model corresponds to similar findings by Umbricht et al. [11] in humans.

45. Picton TW, Hillyard SA, Krausz HI, Galambos R: Human auditory evoked potentials. I: Evaluation of components. Electroencephalogr Clin Neurophysiol 1974, 36:179-190. 